\title{
Supervillin promotes tumor angiogenesis in liver cancer
}

\author{
CHENGGANG ZHAO ${ }^{1,2^{*}}$, ZHIYANG ZHAO ${ }^{1,2^{*}}$, ZHEN WANG $^{1,3^{*}}$, \\ LIZHU HU ${ }^{1,2}$, HONGZHI WANG ${ }^{1,4}$ and ZHIYOU FANG ${ }^{1,4}$
${ }^{1}$ Anhui Province Key Laboratory of Medical Physics and Technology, Center of Medical Physics and Technology,
Hefei Institutes of Physical Science, Chinese Academy of Sciences, Hefei, Anhui 230031;
${ }^{2}$ University of Science and Technology of China, Hefei, Anhui 230026; ${ }^{3}$ Hefei Institute of Stem Cell and
Regenerative Medicine, Guangzhou Institutes of Biomedicine and Health, Chinese Academy of Sciences, Hefei, Anhui 230088; ${ }^{4}$ Department of Pathology and Laboratory Medicine, Hefei Cancer Hospital,
Chinese Academy of Sciences, Hefei, Anhui 230031, P.R. China

Received January 7, 2020; Accepted April 10, 2020

DOI: $10.3892 /$ or.2020.7621

\begin{abstract}
Tumor angiogenesis is a hallmark of liver cancer and is necessary for tumor growth and progression. Supervillin (SVIL) is highly expressed and implicated in several malignant processes of liver cancer. However, the functional relationships between SVIL and tumor angiogenesis in liver cancer have not yet been fully elucidated. The present study was based on bioinformatics analysis, patient tissue sample detection, three-dimensional simulated blood vessel formation, a series of cytological experiments and mouse models. The results-demonstrated the important role of SVIL in the progression of malignant liver cancer and tumor angiogenesis, both in terms of vasculogenic mimicry (VM) and endothelium-dependent vessel (EDV) development. SVIL knockdown inhibited VM formation and induced tumor cell apoptosis via the VEGF-p38 signaling axis and through
\end{abstract}

Correspondence to: Professor Zhiyou Fang, Anhui Province Key Laboratory of Medical Physics and Technology, Center of Medical Physics and Technology, Hefei Institutes of Physical Science, Chinese Academy of Sciences, 350 Shushan Hu Road, Hefei, Anhui 230031, P.R. China

E-mail: z.fang@cmpt.ac.cn

${ }^{*}$ Contributed equally

Abbreviations: COX-2, cyclooxygenase-2; EDV, endotheliumdependent vessel; EMT, epithelial-mesenchymal transition; EphA2, epithelial cell kinase; FAK, focal adhesion kinase; FGF, fibroblast growth factor; HIF-1 $\alpha$, hypoxia-inducible factor-1 $\alpha$; Mig-7, migration-inducing protein 7; MMPs, matrix metalloproteinases; PI3K- $\alpha$, phosphoinositide 3-kinase alpha; SVIL, supervillin; TCGA, The Cancer Genome Atlas; VE-cadherin, vascular endothelial-cadherin; VEGF, vascular endothelium growth factor; $\mathrm{VM}$, vasculogenic mimicry

Key words: supervillin, vascular endothelial growth factor, p38, vascular mimicry, liver cancer various VM-associated transcriptional factors, including vascular endothelial-cadherin, matrix metalloproteinase 9/12 and migration-inducing protein 7. SVIL may therefore be considered a potential tumor vascular biomarker and a promising therapeutic target for patients with liver cancer.

\section{Introduction}

Tumor angiogenesis is a hallmark of liver cancer that is necessary for tumor growth and progression (1-3). Vascular endothelial growth factor (VEGF) is crucial for the development of tumor angiogenesis and exerts its functions by interacting with the tumor microenvironment (4). Drugs targeting the VEGF receptor (VEGFR) for antitumor and anti-angiogenic purposes were considered to be promising for clinical tumor therapy $(4,5)$. However, clinical data have indicated that traditional anti-vascular treatment was limited and vasculogenic mimicry (VM) formation may contribute to tumor development (3).

VM formed by malignant tumor cells serves a role in tumor growth and metastasis, leading to poor patient prognosis (6). The presence of VM may trigger several cascades that increase the availability of oxygen and nutrients from endothelial vessels to tumor tissues, thereby promoting tumor progression (7). The development of vasculogenic mimicry (VM) is similar to that of endothelium-dependent vessels (EDVs) $(6,8)$. Vascular endothelial-cadherin (VE-cadherin), epithelial cell kinase (EphA2), phosphoinositide 3-kinase- $\alpha$ (PI3K- $\alpha$ ), matrix metalloproteinases (MMPs), laminin 5 (Ln-5) $\gamma 2$ chain, hypoxia-inducible factor- $1 \alpha(\mathrm{HIF}-1 \alpha)$, focal adhesion kinase (FAK) and p38 (9-13) are proteins and signaling pathways that promote cell proliferation, migration, invasion and matrix remodeling during VM formation (6). For example, VE-cadherin induces EphA2 phosphorylation by modulating the interaction of EphA2 with its membrane-bound ligand. Additionally, PI3K activation via FAK and ERK1/2-MAPK further mediates MMP14 and MMP2, resulting in VM formation (14-16). In ovarian cancer, the expression of VE-cadherin, EphA2 and MMPs was revealed to be upregulated by VEGF factor A, promoting matrix plasticity and VM formation (17). 
Supervillin (SVIL) is an actin and membrane-associated protein that belongs to the largest sub-family of the villin/gelsolin superfamily $(18,19)$. In tumor cells, SVIL comprises several isoforms, which have been implicated at each step of tumor development, including cell survival, migration and metastasis (20-22). As previously reported, these proteins are involved in cell spreading, lamellipodia extension, actin filament assembly and focal adhesion maturation and/or disassembly (19,23-25). Furthermore, SVIL promotes cancer cell survival by regulating p53 levels (24). A previous study revealed that hypoxia induced an increased SVIL expression, leading to cancer metastasis and poor survival in patients with liver cancer (22). However, the functional relationships between SVIL and tumor angiogenesis/VM formation in liver cancer have not yet been fully elucidated.

\section{Materials and methods}

Cell culture, small interfering (si)RNA transfection and VEGF treatment. Human umbilical vein endothelial cells (HUVECs) were purchased from AllCells LLC (cat. no. H-001F-C) and cultured with HUVEC medium (cat. no. H-004; AllCells LLC) in a $0.25 \%$ gelatin-coated culture flask. HepG2, Bel7405 and MHCC-97H liver cancer cell lines were cultured in DMEM medium (cat. no. C11995; Gibco; Thermo Fisher Scientific, Inc.) and provided by Professor ZY Tang (Liver Cancer Institute, Fudan University, Shanghai, China), which were used in previous studies $(22,26)$. These cell lines were characterized by DNA fingerprinting and isozyme detection. All cell lines used in the present study were regularly authenticated via morphologic observation and tested for the absence of mycoplasma contamination. Samples were last tested for mycoplasma in March 2017.

Cells were transfected with SVIL Stealth siRNA: E4 double stranded (ds)RNA, E5 dsRNA, E11 dsRNA and negative control dsRNA (all Invitrogen; Thermo Fisher Scientific, Inc.; all $40 \mathrm{nM}$ ) using Lipofectamine ${ }^{\circledR}$ RNAi-MAX (Invitrogen; Thermo Fisher Scientific, Inc.). The dsRNA targeting sequences were as follows: E4 dsRNA, 5'-CUCACU UUGAAUGUAGAGAACCAUC-3'; E5 dsRNA, 5'-UUCUGC UGA AGUUAUAGGUUGGGUU-3'; E11 dsRNA, 5'-AGC AUAUUUAGAUUCCUUAUGGCUG-3'.

HepG2 cells were treated with or without $50 \mu \mathrm{g} / 1$ recombinant human VEGF (Novus Biologicals, LLC) at the indicated time-points.

The Cancer Genome Atlas (TCGA) data analysis. The TCGA (https://tcga-data.nci.nih.gov/tcga/) cohort included normal tissues $(n=50)$ and tumor tissues $(n=347)$. The relationship between the expression of genes in liver cancer was analyzed using this database.

Immunohistochemistry (IHC) staining. A liver cancer tissue microarray consisting of 173 pathological samples was purchased from US Biomax, Inc. Liver cancer tissue microarrays were immunohistochemically treated with antibodies against SVIL (dilution 1:1,000; cat. no. S8695; Sigma-Aldrich, Inc.) (24), cluster of differentiation (CD) 31 (dilution 1:1,500; product no. 3528; Cell Signaling Technology, Inc.), CD34 (dilution 1:50; product no. 3569;
Cell Signaling Technology, Inc.), CD146 (dilution 1:500; cat. no. GTX60775; GeneTex, Inc.) and CD248 (dilution 1:500; cat. no. 564993; BD Biosciences). Tissue was sectioned and incubated in $65-75^{\circ} \mathrm{C}$ for $90 \mathrm{~min}$. Samples were then placed in xylene in $25^{\circ} \mathrm{C}$ for $10 \mathrm{~min}$ and re-treated with xylene in $25^{\circ} \mathrm{C}$ for a further $10 \mathrm{~min}$. The sections were sequentially placed in 100, 95 and $80 \%$ ethanol, after which purified water was used for $5 \mathrm{~min}$. Samples were placed into a repair box with antigen repair solution (citrate buffer). A pressure cooker was heated under $1,600 \mathrm{~W}$ to automatically deflate for $2 \mathrm{~min}$ and samples were removed for $2 \mathrm{~min}$ for cooling. The antigen retrieval solution was discarded and sections were rinsed with PBS. The samples were permeabilized with $0.5 \%$ Triton X-100 (prepared in PBS) at room temperature for $20 \mathrm{~min}$. Sections were transferred to a wet box and freshly prepared $3 \%$ hydrogen peroxide was added in $25^{\circ} \mathrm{C}$ for $10 \mathrm{~min}$ to remove endogenous peroxidase blocking solution. Samples were subsequently incubated for $10 \mathrm{~min}$ at room temperature and rinsed with PBS. During washing, slides were immersed in PBS 3 times for 3 min each and blotted dry. Normal goat serum (cat. no. AR0009; Boster Biological Technology co., Ltd) was added dropwise for blocking at room temperature for $30 \mathrm{~min}$. Each slide was dropped with a sufficient quantity of diluted primary antibodies into a wet box and incubated overnight at $4^{\circ} \mathrm{C}$. Rabbit/mouse secondary antibodies (MaxVision TM HRP-Polymer anti-Mouse IHC Kit; cat. no. 5001; MXB Biotechnologies) were subsequently added and incubated for $1 \mathrm{~h}$ at room temperature, after which slides were washed with PBS. DAB was developed for $3 \mathrm{~min}$ in $25^{\circ} \mathrm{C}$ and the degree of staining was assessed under a light microscope (magnification, x100 and x200). Samples were then rinsed again with PBS or tap water for $1 \mathrm{~min}$. The samples were then counterstained with hematoxylin in $25^{\circ} \mathrm{C}$ for $3 \mathrm{~min}$. Samples were subsequently rinsed with tap water for $1 \mathrm{~min}$. Subsequently, dehydration, transparency, sealing, and microscopic examination were performed. The KF-PRO Digital Slide Scanning System (Kongfong Biotech International Co., Ltd.) was used to visualize the resulting signal.

Matrigel tube formation assay. After incubation for $30 \mathrm{~min}$ at $37^{\circ} \mathrm{C}, 2 \times 10^{4}$ cells were added to a 96 -well plate coated with $35 \mu \mathrm{l}$ Matrigel at a concentration of $8.8 \mathrm{mg} / \mathrm{ml}$. Following incubation for $6 \mathrm{~h}$ (liver cancer cells) or at the indicated time-points $\left(0,2,4,6\right.$ and $8 \mathrm{~h}$ ) (HUVEC cells) at $37^{\circ} \mathrm{C}$, three non-overlapping light microscopic images were randomly obtained at low-power magnifications (magnification, x100). Total tube length and the number of branching points formed by endothelial or liver cancer cells per field were measured using Angio Tool 64 0.6a software (National Cancer Institute).

Western blotting. Cells were lysed in RIPA lysis buffer (cat. no. P0013K; Beyotime Institute of Biotechnology) to obtain a protein lysate. Then the protein quantification was determined by BCA. Each $8 \%$ SDS gel was infused with $40 \mu \mathrm{g}$ of protein product. Total protein was separated by SDS-PAGE and transferred to nitrocellulose membranes. Samples were then blocked with $4 \%$ skim milk powder in Tris-buffered saline with Tween-20 (TBST) for $1 \mathrm{~h}$ at room temperature. Primary antibodies were diluted in $4 \%$ skim 
milk powder with TBST and incubated overnight at $4^{\circ} \mathrm{C}$. Membranes were probed with targeted primary antibodies: SVIL (dilution 1:1,000; cat. no. S8695; Sigma-Aldrich, Inc.), $\beta$-tubulin (dilution 1:5,000; cat. no. EM0103; HuaBI), JNK (dilution 1:1,000; product no. 9252; Cell Signaling Technology, Inc.), p-JNK (dilution 1:1,000; product no. 9255; Cell Signaling Technology, Inc.), p38 (dilution 1:1,000; product no. 9212; Cell Signaling Technology, Inc.), p-p38 (dilution 1:1,000; product no. 9211; Cell Signaling Technology, Inc.), VE-cadherin (dilution 1:1,000; product no. 2500; Cell Signaling Technology, Inc.). After washing with TBST three times, the membranes were incubated with horseradish peroxidase-conjugated anti-rabbit IgG antibodies (cat. no. 7074s; Cell Signaling Technology, Inc.) for $1 \mathrm{~h}$ at $25^{\circ} \mathrm{C}$. Proteins were visualized using an ECL luminescent solution (cat. no. 180-5001; Tanon Science and Technology Co., Ltd.).

Cell viability and proliferation assays. For cell viability, cells were seeded in 96-well plates and treated with MTT $(5 \mathrm{mg} / \mathrm{ml})$ for $4 \mathrm{~h}$ at $37^{\circ} \mathrm{C}$. DMSO $(150 \mu \mathrm{l})$ was subsequently added and plates were measured at $450 \mathrm{~nm}$ (CMax Plus; Molecular Devices).

For cell proliferation, cells were cultured in a 12 -well plate at $37^{\circ} \mathrm{C}$. Subsequently, $50 \mu \mathrm{M}$ EdU (cat. no. C10310-3; Guangzhou RiboBio Co., Ltd.) was added for $2 \mathrm{~h}$, after which plates were analyzed according to the manufacturer's protocol. For the BrdU assay, 10-20,000 cells were added per well to a 12 -well plate. After cells reached a density of $30-40 \%$, transient transfection was performed and the culture medium was changed after 4-6 h. Following $48 \mathrm{~h}$ of culture, $10 \mu \mathrm{m}$ BrdU was added to each well plate and incubated for $4 \mathrm{~h}$. Subsequently, the samples were fixed in $4 \%$ paraformaldehyde precooled on ice for $20 \mathrm{~min}$, after which slides were washed three times with PBS. Samples were then further incubated with PBS containing $1.5 \mathrm{~mol} \mathrm{HCl}$ for $10 \mathrm{~min}$ at room temperature and $0.2 \%$ Triton $\mathrm{X}-100$ for $10 \mathrm{~min}$. PBS was used to wash samples in triplicate. BrdU antibodies were diluted to 1:1,000 and $300 \mu \mathrm{l}$ was added to each well. After incubation at $4^{\circ} \mathrm{C}$ overnight, samples were washed three times with PBS and cell nuclei were stained with $0.5 \mu \mathrm{g} / \mathrm{ml}$ DAPI at $25^{\circ} \mathrm{C}$ for $2 \mathrm{~min}$. Distilled water was used to wash off the PBS, glycerin was used to seal the samples and nail polish was sealed around the film.

Cell migration and spreading assays. For cell migration, $2 \times 10^{5} / 100 \mu$ l cells were placed in a Transwell chamber and cultured for $16 \mathrm{~h}$ at $37^{\circ} \mathrm{C}$. Samples were fixed with $4 \%$ paraformaldehyde at $4^{\circ} \mathrm{C}$ for $15 \mathrm{~min}$, stained with $0.1 \%$ crystal violet at $25^{\circ} \mathrm{C}$ for $10 \mathrm{~min}$ and counted using a light microscope (magnification, $\mathrm{x} 100$ ).

To determine the degree of cell spreading, samples were seeded in 12-well plates coated with $10 \mu \mathrm{g} / \mathrm{ml}$ fibronectin and observed at the indicated time-points. Briefly, $800 \mu \mathrm{l}$ of diluted Fibronectin $(10 \mu \mathrm{g} / \mathrm{ml})$ was added to each well of the 12-well plate, and coated at room temperature for $4 \mathrm{~h}$. The transfected cells were digested and resuspended, the cell density was adjusted to $4-5 \times 10^{5}$ cells after counting the cells, and adding them to the coated 12 -well plate. After cells adhered to the wall, cells were observed and images were captured every $30 \mathrm{~min}$.
$V E G F$ release assay. Secreted VEGF was quantified using a VEGF ELISA kit (cat. no. DVE00; R\&D Systems, Inc.). The absorbance of each well was measured at a range of wavelengths based on the manufacturer's protocol. A Microplate ELISA Analyzer (CMax Plus; Molecular Devices, LLC) was used to obtain absorbance data.

Reverse transcription PCR (RT-PCR). HepG2 cells were transiently transfected with siRNA, after which total RNA was extracted using TRIzol ${ }^{\circledR}$ (cat. no. 15596026; Invitrogen; Thermo Fisher Scientific, Inc.). cDNA was subsequently obtained using an RT kit (cat. no. AH311-02; Beijing Transgen Biotech Co., Ltd.) and mRNA levels of associated factors were determined via RT-PCR with cDNA as a template. The reaction was performed in $2 \mathrm{X}$ EasyTaq ${ }^{\circledR}$ PCR SuperMix (cat. no. AS111-11; TransGen Biotech) and the thermocycling conditions were followed according to the instructions. The primer sequences were as follows: 5' to 3': Cyclooxygenase 2 (COX2) forward, ATGATTGCCCGACTCCCTTG and reverse, CCCCACAGCAAACCGTAGAT; EphA2 forward, AGCATCAACCAGACAGAGCC and reverse, AGCATG CCCTTGTACACCTC; N-cadherin forward, CCTGGATCG CGAGCAGATAG and reverse, CCGTGGCTGTGTTTGAAA GG; VE-cadherin forward TCAAGCCCATGAAGCCTC TG and reverse, CCGGTCAAACTGCCCATACT; MMP-2 forward, AACACCTTCTATGGCTGCCC and reverse, GCC GTACTTGCCATCCTTCT; MMP-9 forward, TCCTTATCG CCGACAAGTGG and reverse, AGCGGTCCTGGCAGA AATAG; MMP-12 forward, AACCAACGCTTGCCAAAT CC and reverse, GGCCCGATTCCTTGGAAGTT; MMP-14 forward, ACATCTTCCTGGTGGCTGTG and reverse, GTA CTCGCTATCCACTGCCC; MMP-25 forward, AAGCGA ACCCTGACATGGAG and reverse, CGCCTTCCCATAGAG TTGCT; Mig-7 forward, AGAGGAAAACTGAGGCTGCC and reverse CCGAGTGACAATCTGGGCTT.

In vivo tumorigenicity assays. Healthy nude mice (20 male; age, 3-4 weeks old; weight, 12-14 g) were purchased at the Institute of Model Animal Research of Nanjing University and cultured in an SPF environment. Cells $\left(1 \times 10^{7} / 200 \mu \mathrm{l}\right)$ were injected into the right side of the back of nude mice. After one week, tumor size was measured $\left(\sim 10 \mathrm{~mm}^{3}\right)$, and in vivo SVIL siRNA $(50 \mu \mathrm{l} ; 10 \mathrm{nmol})$ was injected directly into tumor tissue. Injections were administered three times in the first week and then twice a week, for 4 consecutive weeks. The weight of mice and tumor growth was subsequently measured. RNA interference (RNAi) modified with 2'-OMe (Guangzhou RiboBio Co., Ltd.) with the following targeting sequences were utilized: Negative control, 5'-AAUUCUCCGAACGUGUCA CGU-3'; E4 RNAi, 5'-CUCACUUUGAAUGUAGAGAAC CAUC-3'; E5 RNAi, 5'-UUCUGCUGAAGUUAUAGGUUG GGUU-3'; E11 RNAi, 5'-AGCAUAUUUAGAUUCCUUAUG GCUG-3'. Animal experiments were performed according to the guidelines of the Animal Use and Care Committees at Hefei Institutes of Physical Science, CAS.

Statistical analysis. Data are presented as the mean \pm standard deviation. Continuous variables were analyzed using an unpaired Student's t-test for comparisons between two groups. $\mathrm{P}<0.05$ was considered to indicate a statistically significant difference. 
A
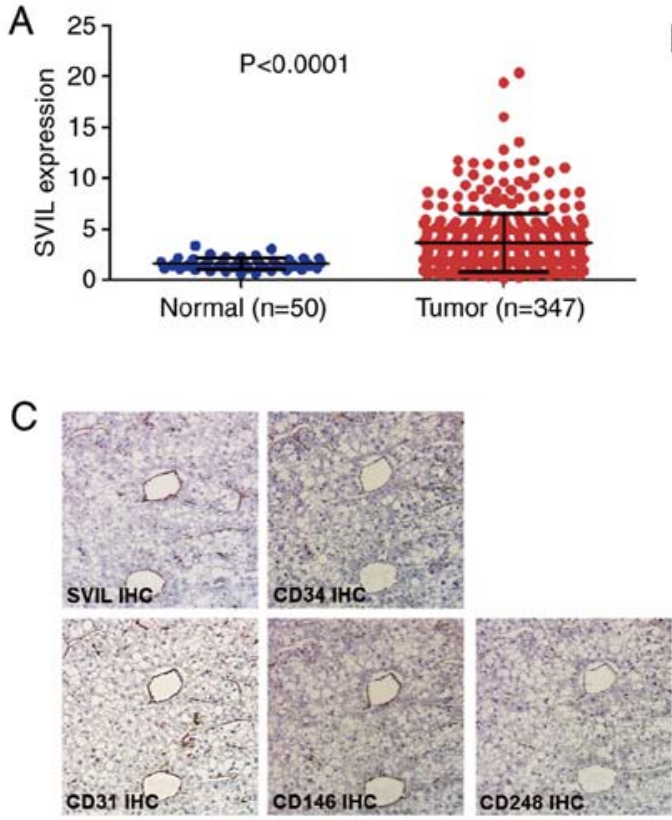

B
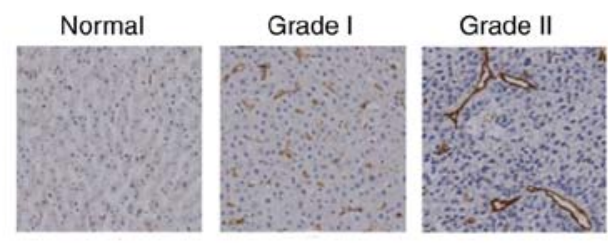

Grade III

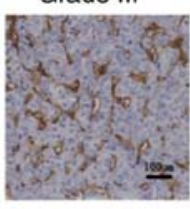

D

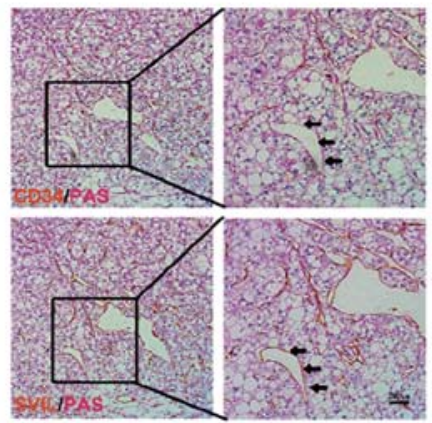

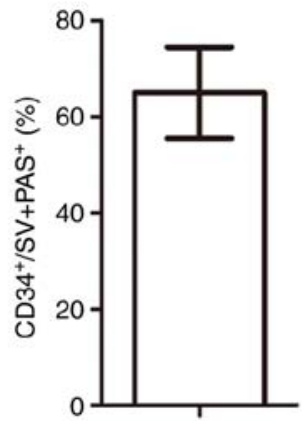

Figure 1. SVIL is highly expressed in liver cancer and localized to new tumor vessels. (A) Analysis of microarray data from The Cancer Genome Atlas. (B) Immunohistochemistry was performed to detect the expression of SVIL in normal liver tissue, grade I, grade II and grade III liver cancer tissue samples (DAB staining; magnification, x100). (C) Liver cancer tissue was serially sectioned and analyzed for SVIL, CD31, CD34, CD146 and CD248 expression (DAB staining; magnification, x100). (D) CD34/PAS staining and SVIL/PAS staining were performed on the same liver cancer tissue area (DAB staining; magnification, $\mathrm{x} 100$ and x200). Statistics of the proportion of CD34-labeled endothelial blood vessels in SVIL-labeled tumor blood vessels. Data are presented as the mean \pm standard deviation. SVIL, supervillin; TCGA, The Cancer Genome Atlas; PAS, periodic acid-Schiff.

\section{Results}

SVIL is upregulated in liver cancer and is localized to tumor vessels. To determine the expression of SVIL in liver cancer tissue, SVIL levels were analyzed in 50 normal liver tissues and 347 liver cancer tissues obtained from The Cancer Genome Atlas (TCGA) database. The results revealed that SVIL was significantly increased in liver cancer compared with normal liver tissue (Fig. 1A). Immunohistochemical staining of liver cancer further indicated that SVIL levels were significantly increased in liver cancer, and positively associated with liver cancer stage (Fig. 1B).

In addition to hepatoma cells, SVIL was expressed in tumor vessels. Tumor vessels of liver cancer primarily include EDV formed via endothelial cells and VM formed via tumor cells $(25,27)$. The expression of SVIL, CD31, CD34, CD146 and CD248 were assessed in serial liver cancer tissue sections via immunohistochemical staining. The results revealed that in the liver cancer samples, certain SVIL-labeled cells were co-located with CD31 and CD34 endothelial cells, exhibiting close proximity to CD146- or CD248-positive pericytes (smooth muscle cells for microvessels; Fig. 1C). Additionally, the results demonstrated that certain SVIL-labeled cells were present on neovascular-like structures formed by non-endothelial cells, presenting as $\mathrm{CD} 34 / \mathrm{PAS}^{+}$and therefore indicating that SVIL may be expressed in vascular mimetic structures (Fig. 1D). The VM structure accounted for $\sim 40 \%$ after determining the number of EDVs labeled with CD34 and the number of tumor blood vessels marked via SVIL (Fig. 1D).

Collectively, the results indicated that SVIL served a role in liver cancer angiogenesis, particularly in EDV and VM development.
SVIL-mediated biological function of endothelial cell promotes endothelium-dependent vessel development. The expression of SVIL was upregulated during angiogenesis in HUVEC cells (Fig. 2A). To determine the potential role of SVIL in HUVEC angiogenesis, Stealth RNAi ${ }^{\mathrm{TM}}$ dsRNAs were used to target sequences within the SVIL coding exon 4 (E4 dsRNA), coding exon 5 (E5 dsRNA), and coding exon 11 (E11 dsRNA). As described previously, each Stealth siRNA that targeted the different axons of SVIL in HUVEC cells reduced the level of each isoform by $\geq 75 \%$. Furthermore, transfection of SVIL-specific RNAi resulted in a $25 \%$ (E4 dsRNA), 40\% (E5 dsRNA) and 55\% (E11 dsRNA) reduction in tube formation during EDV angiogenesis (Figs. 2B and S1A).

The roles of SVIL in HUVEC migration, spread and proliferation were assessed in the present study as these biological functions are important to HUVEC angiogenesis (28). The results revealed that SVIL knockdown inhibited HUVEC migration (Figs. 2C and S1C), spread (Figs. 2D and S1B), viability (Fig. 2E) and proliferation (Fig. 2F), to different degrees. Thus, the results indicated that SVIL served an important role in HUVEC angiogenesis, particularly regarding cell migration, spread and proliferation.

SVIL-mediated biological function of hepatoma carcinoma cells promotes the formation of VM. VM formation may be a primary factor for the failure of traditional anti-vascular treatment (6). The present study therefore analyzed the role of SVIL in the progression of VM development. The results revealed that SVIL expression levels were positively associated with VM formation in liver cancer. This may have been 
A

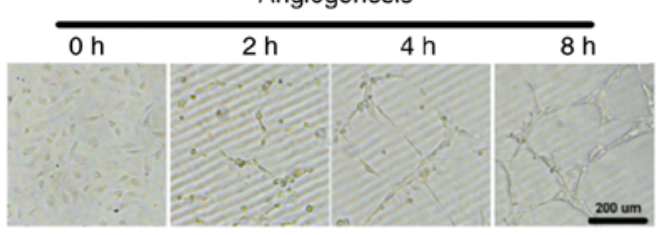

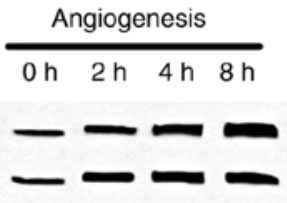

Cell lysis; SVIL IB

Cell lysis; $\beta$-Tubulin IB
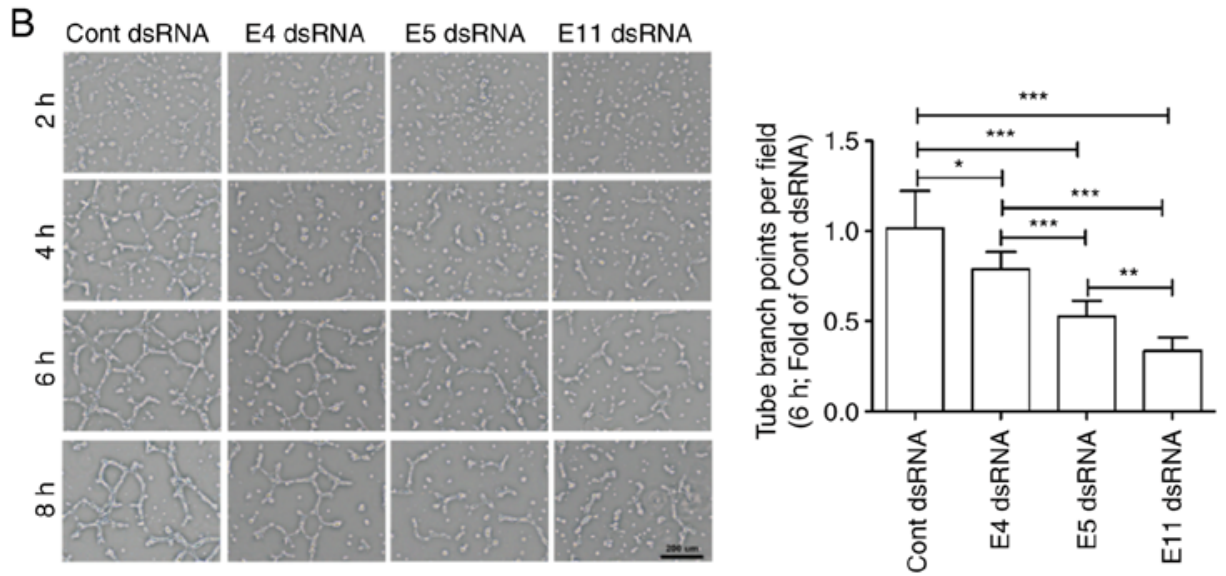

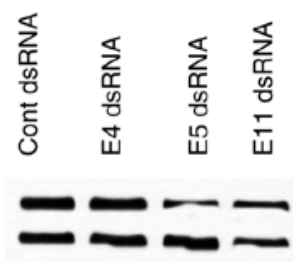

Cell lysis; SVIL IB

Cell Iysis; $\beta$-Tubulin IB
C

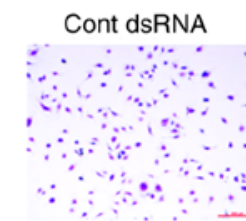

E5 dsRNA

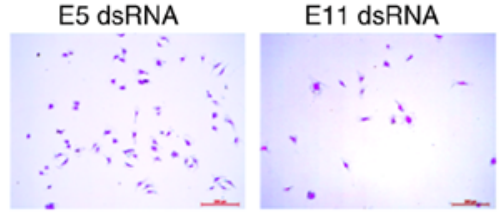

D
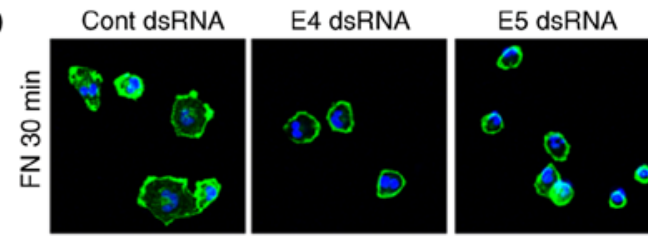

E11 dsRNA
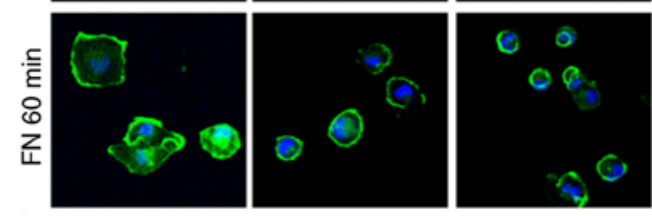

E
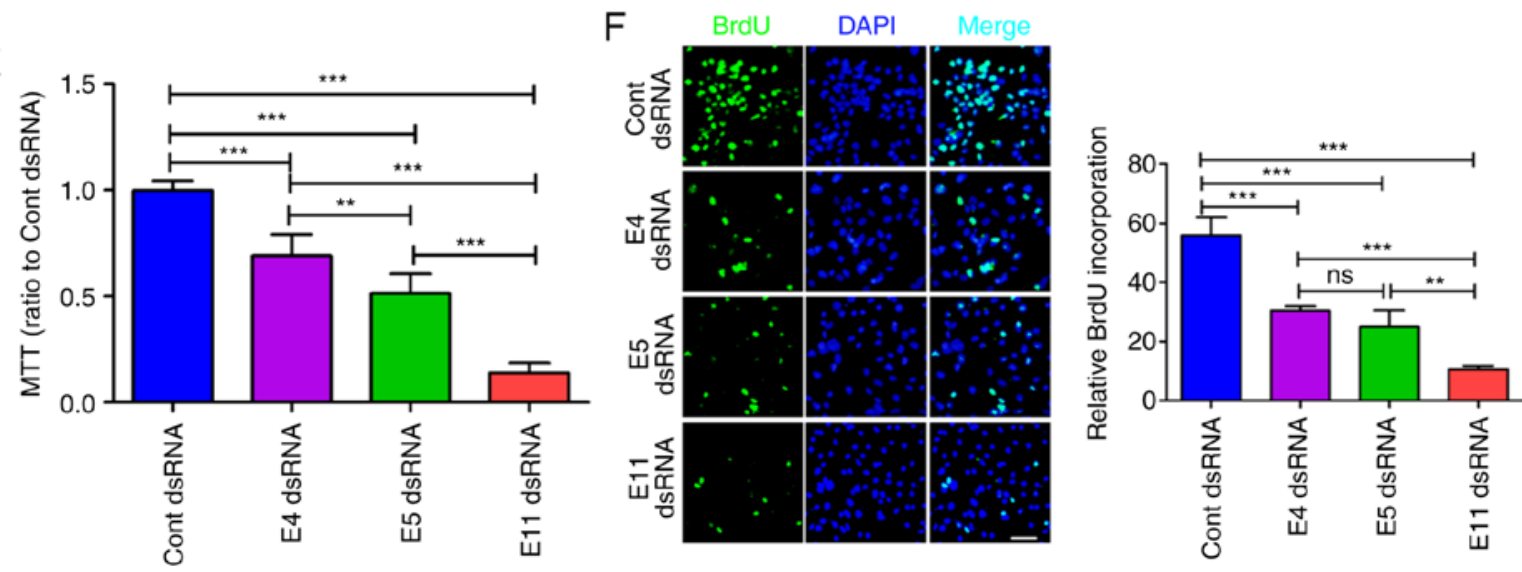

Figure 2. SVIL is positively associated with endothelial cell development and endothelial-dependent angiogenesis. (A) In vitro HUVEC cell tube formation and SVIL expression. (B) HUVEC cell angiogenesis following SVIL knockdown in vitro was observed at 2, 4, 6 and 8 h. The budding rate of HUVEC cells was counted. Western blot analysis was performed to determine the knockout effect of SVIL protein in HUVEC cells. (C) The mobility of HUVEC cells was measured using a Transwell assay following SVIL knockdown. (D) HUVEC cells were transiently transfected with small interfering RNA and seeded into 12-well plates coated with FN. Microfilament staining was then performed to demonstrate cell spreading. (E) An MTT assay was performed to measure cell viability. (F) HUVEC cells with SVIL knockdown were labeled with BrdU to detect cell proliferation. ${ }^{*} \mathrm{P}<0.05$, ${ }^{* *} \mathrm{P}<0.01$ and ${ }^{* * * *} \mathrm{P}<0.001$. Data are presented as the mean \pm standard deviation. SVIL, supervillin; HUVEC, human umbilical vein endothelial cell; FN, fibronectin.

due to HepG2 cells expressing greater quantities of SVIL, gaining a stronger ability to develop VM when compared with other liver cancer cells (Fig. 3A). SVIL knockdown with specific dsRNA resulted in a reduction in VM formation, to 
A

$\beta$-Tubulin

B

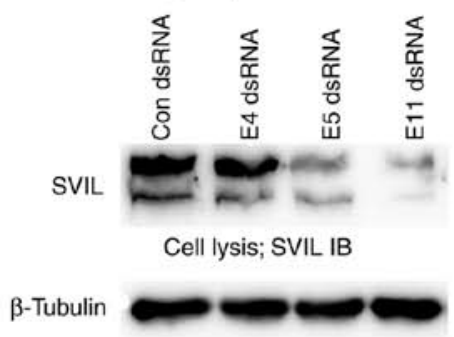

Cell lysis; $\beta$-Tubulin IB
C

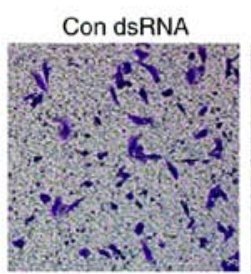

E5 dsRNA

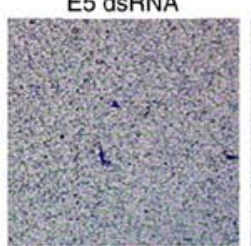

E

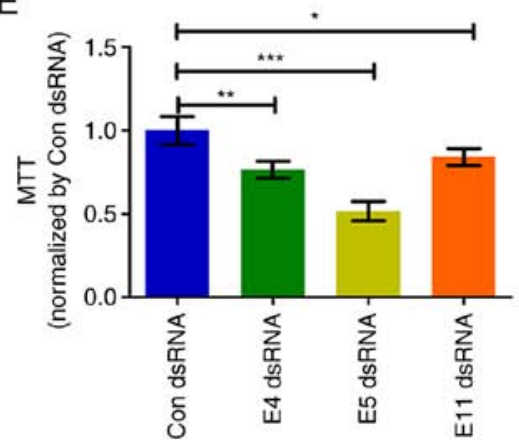

E4 dsRNA

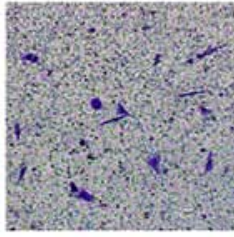

E11 dsRNA

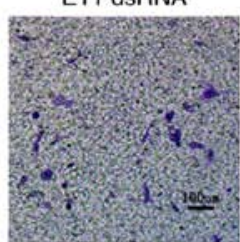

$F$

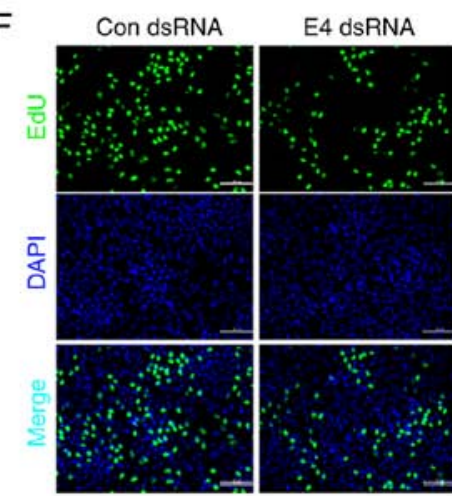

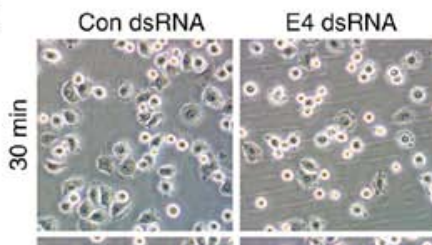

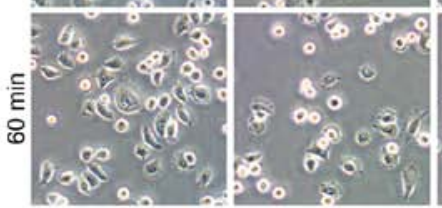

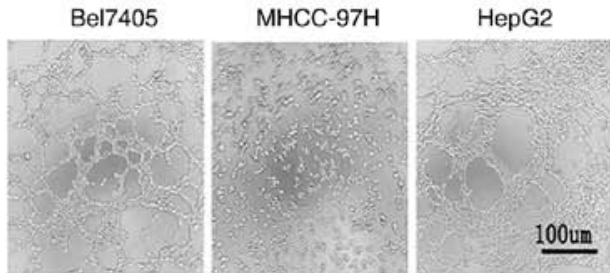

Con dsRnA
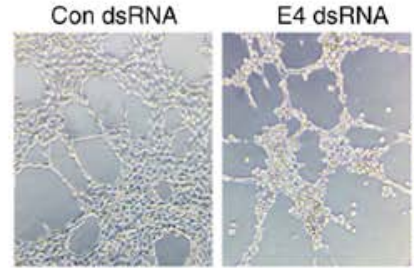

E5 dsRNA
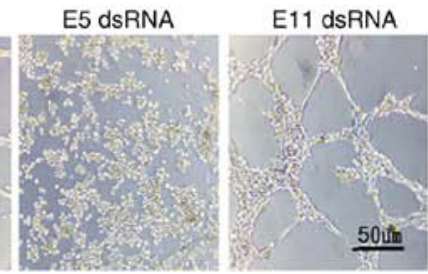

Solin 

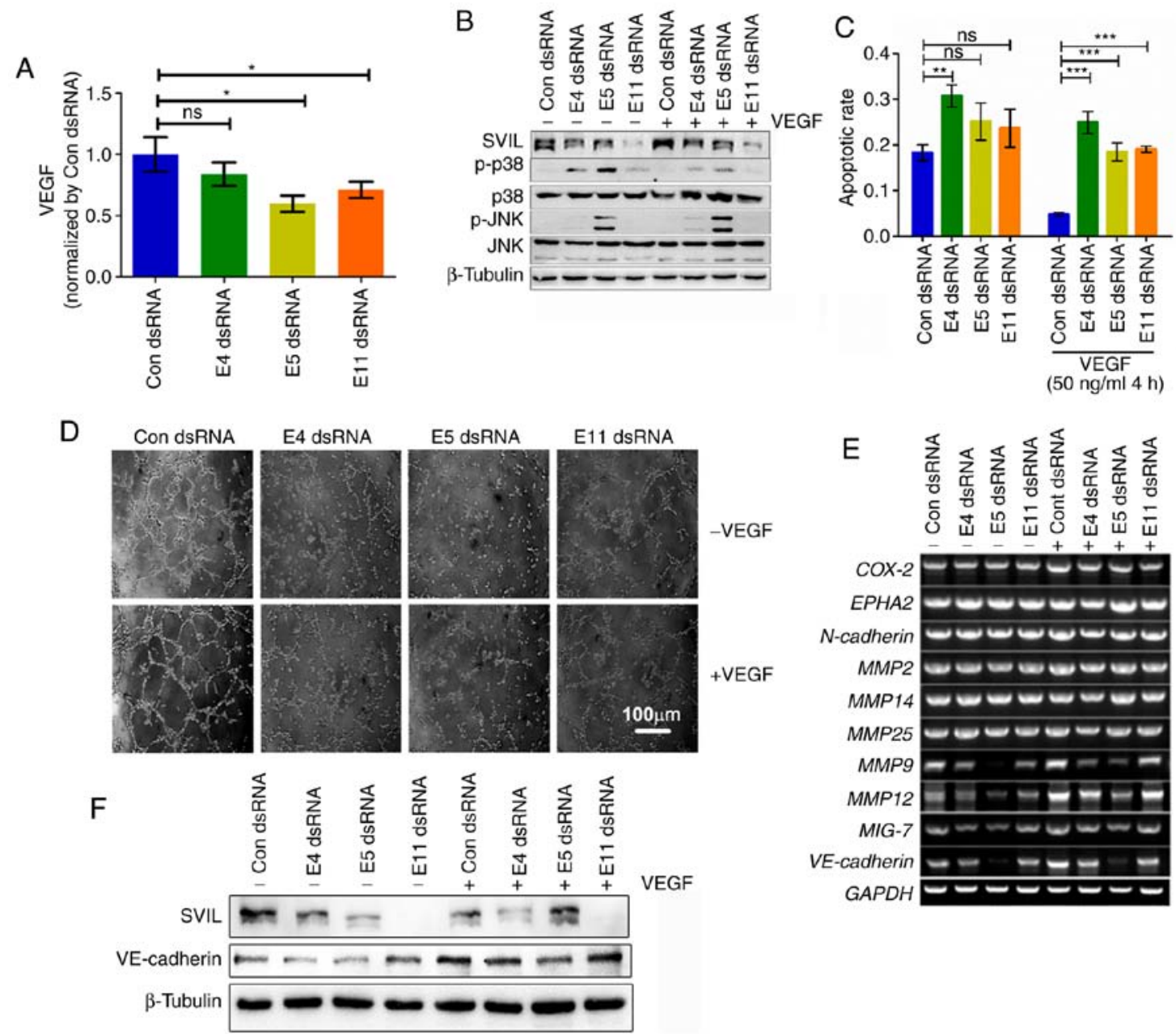

Figure 4. SVIL regulates VM formation via the VEGF/p38 signaling pathway. (A) An ELISA assay was performed to detect the secretion of VEGF in HepG2 cells after SVIL knockown. (B) Western blotting was performed to determine MAPK pathway activation in HepG2 cells with SVIL knockdown and with/without rhVEGF treatment. (C) Apoptosis was detected via flow cytometry. (D) HepG2 cells were knocked down in different isoforms, which were subsequently rescued by VEGF. After transient transfection for $48 \mathrm{~h}$, VEGF (50 ng/ml; $4 \mathrm{~h}$ ) was applied, and the knocked down cells were seeded in Matrigel-coated plates, which were observed under a microscope to determine tube formation. (E) Transcription levels of the relevant factors of VM were determined. (F) Western blotting was performed to detect VE-cadherin levels in HepG2 cells with knocked-down SVIL with/without rhVEGF treatment. ${ }^{*} \mathrm{P}<0.05 ;{ }^{* *} \mathrm{P}<0.01$ and ${ }^{* * *} \mathrm{P}<0.001$. Data are presented as the mean \pm standard deviation. SVIL, supervillin; VM, vasculogenic mimicry; rhVEGF, recombinant human vascular endothelial growth factor.

VM and tumor cells which may lead to tumor progression (Fig. 4A).

The MAPK signaling pathway has been demonstrated to mediate cell survival and migration, and serve an important role in VM formation $(11,30,31)$. It was demonstrated in a previous study that SVIL was associated with MAPK activation $(22,32,33)$. As hypothesized, SVIL knockdown increased p38 activation and the phosphorylation level of JNK compared with the control group (Fig. 4B). Furthermore, cellular apoptosis was increased (Figs. 4C and S3A) and cell population was decreased (Fig. S3B). The results indicated that SVIL may regulate tumor cell survival and p38 activation to ensure VM development.

As VEGF secretion and p38 signaling are indispensable for $\mathrm{VM}$ formation $(6,11,34)$, the present study investigated potential crosstalk between the VEGF and p38 signaling pathways. The results revealed that VEGF abolished the SVIL knockdown-induced reduction of tube formation in VM (Figs. 4D and S4) and downregulated p38 phosphorylation levels. However, the phosphorylation level of JNK did not change, indicating that $\mathrm{p} 38$ phosphorylation may be downstream of SVIL-mediated VEGF secretion (Fig. 4B).

As previously reported, VE-cadherin, N-cadherin, COX-2, EphA2, MMPs and Mig-7 are involved in VM formation $(6,35,36)$. In the present study, SVIL downregulation induced alterations of the VM formation transcriptional network, particularly via VE-cadherin, MMP9, MMP12 and Mig-7. With the addition of VEGF, the expression levels of these transcriptional factors were rescued by SVIL knockdown (Fig. 4E and F). Collectively, the results suggested that SVIL promoted VM development by activating the VEGF-p38 axis and inducing VM-associated transcriptional factors.

SVIL knockdown suppresses liver tumor growth in vivo. As tumor angiogenesis promotes the proliferation, growth, invasion and metastasis of hepatoma cells $(1,2,37)$, the present study investigated whether SVIL knockdown inhibited tumor growth in vivo. Hepatoma cells were injected into the right side of the back of each nude mouse. After a week, in vivo SVIL siRNA was injected directly into the tumor tissue. The 
A

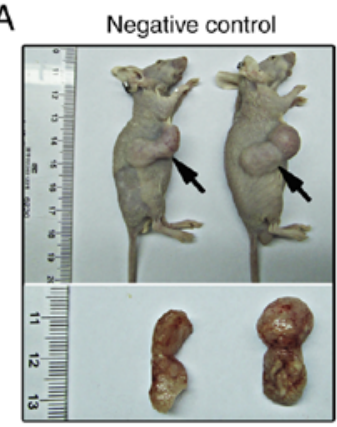

B

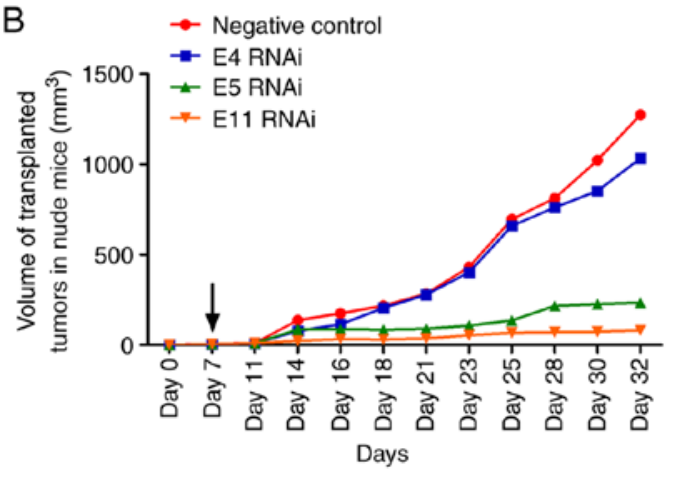

E4 RNAi

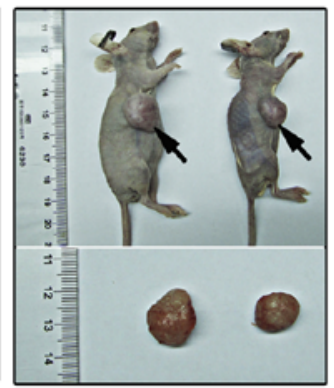

C
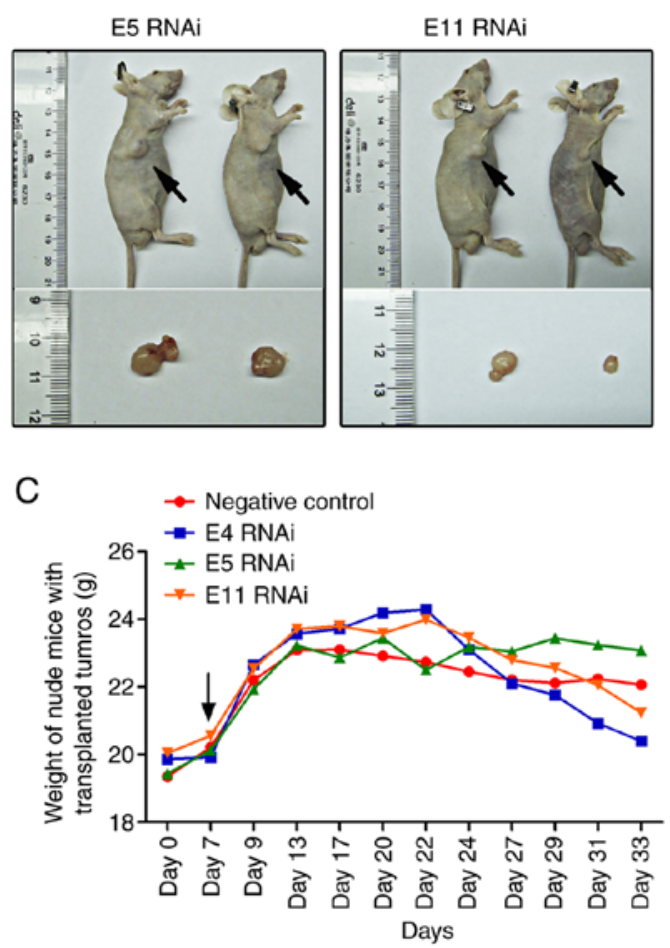

Figure 5. SVIL knockdown inhibits tumor growth in vivo. (A) Male nude mice BALB/C-nu with tumors were untreated or treated in vivo with SVIL knockdown $(n=5)$. (B) The volume of transplanted tumors in nude mice were determined. The E4 knockdown isoform of SVIL exhibited a similar trend to the negative control. However, in the other two groups, the tumor volume was significantly inhibited from the 14th day. (C) Differences in body weights were observed in each group. SVIL, supervillain.

results revealed that injection of in vivo SVIL siRNA significantly inhibited tumor growth, particularly E5 RNAi and E11 RNAi (Fig. 5A and B). However, mouse weight did not differ compared with the negative control RNAi-treated mice (Fig. 5C).

\section{Discussion}

Liver cancer is a solid malignant tumor with a dense vascular network, a high level of metastasis, high recurrence and poor prognosis (38). It has been revealed that tumor angiogenesis serves an important role in the proliferation, growth, invasion and metastasis of hepatoma cells. Recent studies have focused on the inhibition of tumor angiogenesis and the development of small-molecule targeted drugs to inhibit angiogenesis (39-41). There are two major forms of tumor angiogenesis during tumorigenesis: EDV and VM. EDV is composed of endothelial cells, while VM is constructed via in situ cancer cells. SVIL is an actin-associated protein that is involved in various cell movements, such as adhesion and migration (41). A previous study revealed that SVIL promoted the malignant progression of liver cancer (22). Therefore, the present study investigated whether SVIL was associated with EDV and VM. The results indicated that SVIL promoted EDV development and induced VM formation. Furthermore, decreased SVIL ultimately inhibited VM formation by suppressing VEGF secretion to activate p38-induced tumor cell apoptosis (Fig. 6).

The results of the present study revealed that SVIL was highly expressed in liver cancer compared with normal liver tissues and localized to tumor neovascular sites. Additionally, VM accounted for $\sim 40 \%$ of the total number of new blood

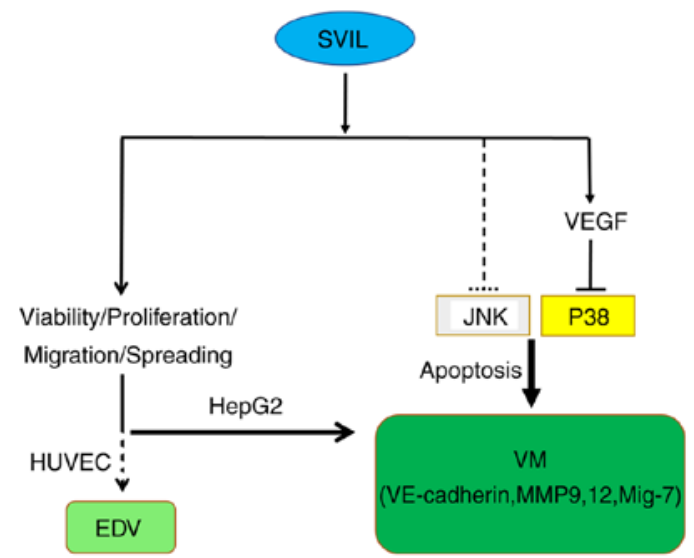

Figure 6. Diagram of the pathways associated with liver cancer angiogenesis (VM and EDV) that are affected by SVIL. $\downarrow$ indicates acceleration and $\perp_{\text {indi- }}$ cates suppression. VM, vasculogenic mimicry; EDV, endothelium-dependent vessel; SVIL, supervillin.

vessels, indicating the significance of VM in tumor formation. Further studies revealed that SVIL expression was closely associated with the migration, spread, viability and proliferation of endothelial cells, as well as tumor cells in liver cancer. Changes in biological function affected the formation of new blood vessels. SVIL was revealed to serve an important role in each step of tumor development, including cell survival, migration and metastasis. SVIL was revealed to also regulate tumor cell survival by inhibiting the ubiquitin-specific-processing protease 7-dependent deubiquitination of p53 (24). SVIL has also been revealed to serve an important role in cell migration 
and invasion (21). In podosomes and invadopodia, SVIL was revealed to recombine the cortical actin cytoskeleton, promote the formation of podosomes and invadopodia, and promote ECM degradation, thereby inducing tumor cell motility $(42,43)$.

The MAPK is involved in the neovascularization process, and the ERK/JNK/p38-MAPK pathways separately/co-executively perform positive or negative functions (44). In a previous study, it was determined that SVIL promoted invasion, metastasis, and epithelial-mesenchymal transition (EMT) processes by activating the RhoA/ROCK-ERK/p38 signaling pathway during hypoxia (22). However, under normoxic conditions, reduced SVIL may activate the p38 signaling pathway and cell apoptosis, which may be accompanied by JNK activation. It has been reported that the p38 inhibitor, SB202190, induces angiogenesis by reducing apoptosis, thereby increasing DNA synthesis and cell proliferation, and enhancing cell differentiation, which involves fibroblast growth factor (FGF)-2 (44). In the present study, SVIL knockdown significantly suppressed VEGF secretion in HepG2 cell culture media. VEGF treatment also inhibited p38, but not the JNK, pathway and partially rescued VM formation, suggesting that SVIL regulated VM formation via the VEGF-p38 axis. VEGF was secreted from tumor cells, which may, in turn, promote tumor metastasis and angiogenesis. This may partly explain the role of SVIL in endothelial cell angiogenesis.

$\mathrm{VM}$ formation is a complex process that involves a variety of pathways and signaling molecules, including factors associated with tumor cell invasion, migration, apoptosis and matrix remodeling, such as VE-cadherin, $\mathrm{N}$-cadherin, EphA2, MMPs and COX-2 (6). The results of the present study revealed that changes in VE-cadherin, MMP9, MMP12 and Mig-7 levels were positively associated with SVIL and recovered following VEGF treatment. Transfection of E5 dsRNA demonstrated more significant alterations in VE-cadherin, MMP9, MMP12 and Mig-7 levels when compared with E4 and E11 dsRNA. This may be dependent on the ratio of the three isoforms in tumor cells and the differences in dsRNA levels in target sites. Among the aforementioned molecules, VE-cadherin is an important adhesion protein. VE-cadherin regulates EphA2, which is an important factor in VM formation $(45,46)$. The p38/MAPK signal that occurs during VE-cadherin regulation often regulates cell membrane permeability and cytoskeletal remodeling (47). p38 inhibition, but not ERK-MAPK, was revealed to significantly reduce the loss of membrane-associated VE-cadherin $(48,49)$. MMPs are vital for the degradation and integration of the matrix. Twist1 was revealed to promote MMP2 and MMP9 activation, thereby inducing liver cancer invasion during the EMT process (50). In addition, epigallocatechin-3-gallate inhibited pancreatic tumor cell growth, invasion, angiogenesis and metastasis. This involved promoting p38 and JNK activities and significantly reducing MMP9 and MMP12 (51). Mig-7 is overexpressed in highly invasive melanoma and invasive melanoma conversely, and it increases the Ln-5 22 chain domain III fragment, thereby promoting tumor cell migration, migration and VM formation (52). Collectively, the results of the present study indicated that SVIL promoted VM development by activating the VEGF/p38 axis and inducing $\mathrm{VM}$-associated transcriptional factors.
In summary, the results elucidated the important role of SVIL in the progression of malignant liver cancer and tumor angiogenesis, both in EDV and VM. Particularly, decreased SVIL levels inhibited VM formation by reducing VEGF secretion to activate the p38 pathway and regulate VE-cadherin/MMP9/12/Mig-7 transcription. As a result, SVIL may be considered as a potential tumor vascular biomarker and a promising therapeutic target for patients with liver cancer.

\section{Acknowledgments}

We would like to thank the members of the technical assistance team at the Center of Medical Physics and Technology, Hefei Institutes of Physical Science.

\section{Funding}

The present study was supported by the National Natural Science Foundation of China (grant. nos. 31571433, 81773131, and 81872066), the Anhui Provincial Natural Science Foundation (grant. nos. 1608085MH180 and 1808085QH272), the Innovative program of the Development Foundation of Hefei Center for Physical Science and Technology (grant. no. 2017FXCX008), and the CASHIPS Director's Fund (grant. no. YZJJ201704).

\section{Availability of data and materials}

The datasets used and/or analyzed during the current study are available from the corresponding author on reasonable request.

\section{Authors' contributions}

$\mathrm{ZF}, \mathrm{CZ}$, and ZW conceived and designed the article. ZW, CZ and ZZ, jointly completed cytology experiments and histological examination of liver specimens. The manuscript was written by $\mathrm{CZ}$ and critically reviewed by all participating authors. The data collection and statistical analysis were performed by $\mathrm{ZZ}$, LH, and HW. All authors have read and approved the final manuscript and agree to be accountable for all aspects of the research in ensuring that the accuracy or integrity of any part of the work are appropriately investigated and resolved.

\section{Ethics approval and consent to participate}

Study protocols were approved by the Institutional Review Board of the Cancer Hospital of Hefei Institutes of Physical Science, Chinese Academy of Sciences (CAS). Animal experiments were performed according to the guidelines of the Animal Use and Care Committees at Hefei Institutes of Physical Science, CAS.

\section{Patient consent for publication}

Not applicable.

\section{Competing interests}

The authors declare that they have no competing interests related to this work. 


\section{References}

1. Zhu AX, Duda DG, Sahani DV and Jain RK: HCC and angiogenesis: Possible targets and future directions. Nat Rev Clin Oncol 8: 292-301, 2011

2. Fernandez M, Semela D, Bruix J, Colle I, Pinzani M and Bosch J: Angiogenesis in liver disease. J Hepatol 50: 604-620, 2009.

3. Wang Z, Dabrosin C, Xin Y,Fuster MM, Arreola A, Rathmell WK, Generali D, Nagaraju GP, El-Rayes B, Ribatti D, et al: Broad targeting of angiogenesis for cancer prevention and therapy. Semin Cancer Biol 35 (Suppl): S224-S243, 2015.

4. Hicklin DJ and Ellis LM: Role of the vascular endothelial growth factor pathway in tumor growth and angiogenesis. J Clin Oncol 23: 1011-1027, 2005.

5. Viallard C and Larrivée B: Tumor angiogenesis and vascular normalization: Alternative therapeutic targets. Angiogenesis 20: 409-426, 2017

6. Qiao L, Liang N, Zhang J, Xie J, Liu F, Xu D, Yu X and Tian Y: Advanced research on vasculogenic mimicry in cancer. J Cell Mol Med 19: 315-326, 2015.

7. Döme B, Hendrix MJ, Paku S, Tóvári J and Tímár J: Alternative vascularization mechanisms in cancer: Pathology and therapeutic implications. Am J Pathol 170: 1-15, 2007.

8. Wang W, Lin P, Sun BC, Cai WJ, Han CR, Li L, Lu HH and Zhang JM: Role of vasculogenic mimicry and endothelium-dependent vessel in metastasis of laryngeal cancer. Zhonghua Er Bi Yan Hou Tou Jing Wai Ke Za Zhi 47: 400-405, 2012 (In Chinese).

9. Elpek GÖ: Angiogenesis and liver fibrosis. World J Hepatol 7: 377-391, 2015.

10. Delgado-Bellido D, Serrano-Saenz S, Fernández-Cortés M and Oliver FJ: Vasculogenic mimicry signaling revisited: Focus on non-vascular VE-cadherin. Mol Cancer 16: 65, 2017.

11. Ling G, Ji Q, Ye W, Ma D and Wang Y: Epithelial-mesenchymal transition regulated by $\mathrm{p} 38 / \mathrm{MAPK}$ signaling pathways participates in vasculogenic mimicry formation in SHG44 cells transfected with TGF- $\beta$ cDNA loaded lentivirus in vitro and in vivo. Int J Oncol 49: 2387-2398, 2016.

12. Wang H, Lin H, Pan J, Mo C, Zhang F, Huang B, Wang Z, Chen X, Zhuang J, Wang D and Qiu S: Vasculogenic mimicry in prostate cancer: The roles of EphA2 and PI3K. J Cancer 7: 1114-1124, 2016.

13. Trapani F, Metcalf RL, Polanski R, Fusi A, Hodgkinson C, Nonaka D, Hendrix MJ, Morrrow C, Blackhall F, Simpson KL and Dive C: 264 Vasculogenic mimicries in small cell lung cancer. European J Cancer 50 (Suppl 6): S88, 2014.

14. Brantley DM, Cheng N, Thompson EJ, Lin Q, Brekken RA, Thorpe PE, Muraoka RS, Cerretti DP, Pozzi A, Jackson D, et al: Soluble Eph A receptors inhibit tumor angiogenesis and progression in vivo. Oncogene 21: 7011-7026, 2002.

15. Hess AR and Hendrix MJ: Focal adhesion kinase signaling and the aggressive melanoma phenotype. Cell Cycle 5: 478-480, 2006.

16. Cheng N, Brantley DM, Liu H, Lin Q, Enriquez M, Gale N, Yancopoulos G, Cerretti DP, Daniel TO and Chen J: Blockade of EphA receptor tyrosine kinase activation inhibits vascular endothelial cell growth factor-induced angiogenesis. Mol Cancer Res 1: 2-11, 2002.

17. Wang JY, Sun T, Zhao XL, Zhang SW, Zhang DF, Gu Q, Wang XH, Zhao N, Qie S and Sun BC: Functional significance of VEGF-a in human ovarian carcinoma: Role in vasculogenic mimicry. Cancer Biol Ther 7: 758-766, 2008.

18. Chen Y, Takizawa N, Crowley JL, Oh SW, Gatto CL, Kambara T, Sato O, Li XD, Ikebe M and Luna EJ: F-actin and myosin II binding domains in supervillin. J Biol Chem 278: 46094-46106, 2003.

19. Pestonjamasp KN, Pope RK, Wulfkuhle JD and Luna EJ: Supervillin (p205): A novel membrane-associated, F-actinbinding protein in the villin/gelsolin superfamily. J Cell Biol 139: $1255-1269,1997$.

20. Oh SW, Pope RK, Smith KP, Crowley JL, Nebl T, Lawrence JB and Luna EJ: Archvillin, a muscle-specific isoform of supervillin, is an early expressed component of the costameric membrane skeleton. J Cell Sci 116: 2261-2275, 2003.

21. Chen X, Yang H, Zhang S, Wang Z, Ye F, Liang C, Wang H and Fang Z: A novel splice variant of supervillin, SV5, promotes carcinoma cell proliferation and cell migration. Biochem Biophys Res Commun 482: 43-49, 2017.
22. Chen X, Zhang S, Wang Z, Wang F, Cao X, Wu Q, Zhao C, Ma H, Ye F, Wang $\mathrm{H}$ and Fang Z: Supervillin promotes epithelialmesenchymal transition and metastasis of hepatocellular carcinoma in hypoxia via activation of the RhoA/ROCK-ERK/p38 pathway. J Exp Clin Cancer Res 37: 128, 2018.

23. Takizawa N, Ikebe R, Ikebe M and Luna EJ: Supervillin slows cell spreading by facilitating myosin II activation at the cell periphery. J Cell Sci 120: 3792-3803, 2007.

24. Fang $\mathrm{Z}$ and Luna EJ: Supervillin-mediated suppression of p53 protein enhances cell survival. J Biol Chem 288: 7918-7929, 2013.

25. Jue C, Zhifeng W, Zhisheng Z, Lin C, Yayun Q, Feng J, Hao G, Shintaro I, Hisamitsu T, Shiyu G and Yanqing L: Vasculogenic mimicry in hepatocellular carcinoma contributes to portal vein invasion. Oncotarget 7: 77987-77997, 2016.

26. Ye QH, Zhu WW, Zhang JB, Qin Y, Lu M, Lin GL, Guo L, Zhang B, Lin ZH, Roessler S, et al: GOLM1 modulates EGFR/RTK cell-surface recycling to drive hepatocellular carcinoma metastasis. Cancer Cell 30: 444-458, 2016.

27. Gorrin-Rivas MJ, Arii S, Mori A, Takeda Y, Mizumoto M, Furutani $\mathrm{M}$ and Imamura M: Implications of human macrophage metalloelastase and vascular endothelial growth factor gene expression in angiogenesis of hepatocellular carcinoma. Ann Surg 231: 67-73, 2000.

28. Lamouille S, Mallet C, Feige JJ and Bailly S: Activin receptor-like kinase 1 is implicated in the maturation phase of angiogenesis. Blood 100: 4495-4501, 2002.

29. Wu HB, Yang S, Weng HY, Chen Q, Zhao XL, Fu WJ, Niu Q, Ping YF, Wang JM, Zhang X, et al: Autophagy-induced KDR/VEGFR-2 activation promotes the formation of vasculogenic mimicry by glioma stem cells. Autophagy 13: 1528-1542, 2017.

30. Azad T, Janse van Rensburg HJ, Lightbody ED, Neveu B, Champagne A, Ghaffari A, Kay VR, Hao Y, Shen H, Yeung B, et al: A LATS biosensor screen identifies VEGFR as a regulator of the Hippo pathway in angiogenesis. Nat Commun 9: 1061,2018

31. Paulis YWJ, Dinnes D, Soetekouw PMMB, Nelson PJ, Burdach S, Loewe RP, Tjan-Heijnen VCG, von Luettichau I and Griffioen AW: Imatinib reduces the vasculogenic potential of plastic tumor cells. Current Angiogenesis 1: 64-71, 2012.

32. Fang Z, Takizawa N, Wilson KA, Smith TC, Delprato A, Davidson MW, Lambright DG and Luna EJ: The membrane-associated protein, supervillin, accelerates F-actin-dependent rapid integrin recycling and cell motility. Traffic 11: 782-799, 2010.

33. Liu HP, Yu MC, Jiang MH, Chen JX, Yan DP, Liu F and Ge BX: Association of supervillin with KIR2DL1 regulates the inhibitory signaling of natural killer cells. Cell Signal 23: 487-496, 2011.

34. Bai XL, Zhang Q, Ye LY, Liang F, Sun X, Chen Y, Hu QD, Fu QH, $\mathrm{Su} \mathrm{W}$, Chen Z, et al: Myocyte enhancer factor $2 \mathrm{C}$ regulation of hepatocellular carcinoma via vascular endothelial growth factor and Wnt/ $\beta$-catenin signaling. Oncogene 34: 4089-4097, 2015.

35. Ren K, Zhang J, Gu X, Wu S, Shi X, Ni Y, Chen Y, Lu J, Gao Z, Wang $C$ and Yao N: Migration-inducing gene-7 independently predicts poor prognosis of human osteosarcoma and is associated with vasculogenic mimicry. Exp Cell Res 369: 80-89, 2018.

36. Folberg R, Hendrix MJ and Maniotis AJ: Vasculogenic mimicry and tumor angiogenesis. Am J Pathol 156: 361-381, 2000.

37. Yang $\mathrm{C}, \mathrm{Xu} \mathrm{Y}$, Cheng $\mathrm{F}, \mathrm{Hu} \mathrm{Y}$, Yang $\mathrm{S}$, Rao J and Wang $\mathrm{X}$ : MiR-1301 inhibits hepatocellular carcinoma cell migration, invasion, and angiogenesis by decreasing Wnt/ $/$-catenin signaling through targeting BCL9. Cell Death Dis 8: e2999, 2017.

38. Colombo M: Liver cancer. Lancet 3: 55, 1995.

39. Mashreghi M, Azarpara H, Bazaz MR, Jafari A, Masoudifar A, Mirzaei H and Jaafari MR: Angiogenesis biomarkers and their targeting ligands as potential targets for tumor angiogenesis. J Cell Physiol 233: 2949-2965, 2018.

40. Niccoli Asabella A, Di Palo A, Altini C, Ferrari C and Rubini G: Multimodality imaging in tumor angiogenesis: Present status and perspectives. Int J Mol Sci 18: E1864, 2017.

41. Casazza A, Fu X, Johansson I, Capparuccia L, Andersson F, Giustacchini A, Squadrito ML, Venneri MA, Mazzone M, Larsson E, et al: Systemic and targeted delivery of semaphorin $3 \mathrm{~A}$ inhibits tumor angiogenesis and progression in mouse tumor models. Arterioscler Thromb Vasc Biol 31: 741-749, 2011.

42. Crowley JL, Smith TC, Fang Z, Takizawa N and Luna EJ: Supervillin reorganizes the actin cytoskeleton and increases invadopodial efficiency. Mol Biol Cell 20: 948-962, 2009. 
43. Gimona M, Buccione R, Courtneidge SA and Linder S: Assembly and biological role of podosomes and invadopodia. Curr Opin Cell Biol 20: 235-241, 2008.

44. Matsumoto T, Turesson I, Book M, Gerwins $P$ and Claesson-Welsh L: p38 MAP kinase negatively regulates endothelial cell survival, proliferation, and differentiation in FGF-2-stimulated angiogenesis. J Cell Biol 156: 149-160, 2002.

45. Kirschmann DA, Seftor EA, Hardy KM, Seftor RE and Hendrix MJ: Molecular pathways: Vasculogenic mimicry in tumor cells: Diagnostic and therapeutic implications. Clin Cancer Res 18: 2726-2732, 2012.

46. Hess AR, Seftor EA, Gruman LM, Kinch MS, Seftor RE and Hendrix MJ: VE-cadherin regulates EphA2 in aggressive melanoma cells through a novel signaling pathway: Implications for vasculogenic mimicry. Cancer Biol Ther 5: 228-233, 2006.

47. Tremblay PL, Auger FA and Huot J: Regulation of transendothelial migration of colon cancer cells by E-selectin-mediated activation of p38 and ERK MAP kinases. Oncogene 25: 6563-6573, 2006.

48. Nwariaku FE, Chang J, Zhu X, Liu Z, Duffy SL, Halaihel NH, Terada $\mathrm{L}$ and Turnage RH: The role of $\mathrm{p} 38$ map kinase in tumor necrosis factor-induced redistribution of vascular endothelial cadherin and increased endothelial permeability. Shock 18 : 82-85, 2002.
49. Kimura T, Mogi C, Sato K, Tomura H, Ohta H, Im DS, Kuwabara A, Kurose H, Murakami M and Okajima F: p2y5/LPA(6) attenuates LPA(1)-mediated VE-cadherin translocation and cell-cell dissociation through $\mathrm{G}(12 / 13)$ protein-Src-Rap1. Cardiovasc Res 92: 149-158, 2011.

50. Zhao XL, Sun T, Che N, Sun D, Zhao N, Dong XY, Gu Q, Yao Z and Sun BC: Promotion of hepatocellular carcinoma metastasis through matrix metalloproteinase activation by epithelial-mesenchymal transition regulator Twist1. J Cell Mol Med 15: 691-700, 2011.

51. Shankar S, Ganapathy S, Hingorani SR and Srivastava RK: EGCG inhibits growth, invasion, angiogenesis, and metastasis of pancreatic cancer. Front Biosci 13: 440-452, 2008.

52. Petty AP, Garman KL, Winn VD, Spidel CM and Lindsey JS: Overexpression of carcinoma and embryonic cytotrophoblast cell-specific Mig-7 induces invasion and vessel-like structure formation. Am J Pathol 170: 1763-1780, 2007.

This work is licensed under a Creative Commons Attribution-NonCommercial-NoDerivatives 4.0 International (CC BY-NC-ND 4.0) License. 